\title{
Classification of Non-ferrous Scrap Metal using Two Component Magnetic Induction Spectroscopy
}

Link to publication record in Manchester Research Explorer

Citation for published version (APA):

O'Toole, M. D., \& Peyton, A. J. (2019). Classification of Non-ferrous Scrap Metal using Two Component Magnetic Induction Spectroscopy.

\section{Citing this paper}

Please note that where the full-text provided on Manchester Research Explorer is the Author Accepted Manuscript or Proof version this may differ from the final Published version. If citing, it is advised that you check and use the publisher's definitive version.

\section{General rights}

Copyright and moral rights for the publications made accessible in the Research Explorer are retained by the authors and/or other copyright owners and it is a condition of accessing publications that users recognise and abide by the legal requirements associated with these rights.

\section{Takedown policy}

If you believe that this document breaches copyright please refer to the University of Manchester's Takedown Procedures [http://man.ac.uk/04Y6Bo] or contact uml.scholarlycommunications@manchester.ac.uk providing relevant details, so we can investigate your claim.

\section{OPEN ACCESS}




\title{
Classification of Non-ferrous Scrap Metal using Two Component Magnetic Induction Spectroscopy
}

\author{
M. D. O'Toole and A. J. Peyton \\ School of Electrical and Electronic Engineering \\ The University of Manchester \\ Manchester, M13 9PL, UK \\ Email: michael.otoole@manchester.ac.uk
}

\begin{abstract}
Magnetic induction spectroscopy is the measurement of how a conductive object reflects and scatters a magnetic field over different frequencies in response to some excitation magnetic field. In recent work, we proposed using this technique to classify different non-ferrous metals for the recycling and resource recovery sector - specifically, to identify fragments of scrap aluminium, copper and brass in shredded waste streams for separation and recovery. We proposed a simple algorithm that used only two components of the spectra that gave strong purity and recovery-rates when tested on a manufactured control set cut from stock metals.

In this paper, we re-examined this method using real scrap metal samples drawn from a commercial sorting line. We found moderate purity and recovery-rates of brass and copper of between around $70 \%$ and $90 \%$. However, the classification of aluminium was poor with $\approx \mathbf{5 5} \%$ and $\approx \mathbf{8 0} \%$ purity and recovery rates respectively. Magnetic induction sensors are a natural fit for the specifications of the industry. They are capable of highthroughputs, are unaffected by dirt or contaminants and are mechanically and physically robust. Although our results are modest, they are not insignificant given the simplicity of the algorithm and the relatively low-cost of instrumentation. Our work suggests the MIS as a technique may have a significant role to play in the extraction and recovery of non-ferrous resources.
\end{abstract}

\section{INTRODUCTION}

The global demand for consumer products in the face of finite resources poses a significant challenge to our future prosperity. It has driven a focus on sustainable economic model,s such as the Circular Economy where waste resources are recycled to become the feedstock and raw material of the new. There is a political will in some quarters to encourage such approaches, signified for example by legislative targets such as European Union issuing directive 2000/53/EC, imposing an $85 \%$ recycle and reuse rate for End-of-Life Vehicles (ELVs) by 2015 [1]. Each year, over 40 million vehicles are designated end-of-life and scrapped worldwide, nearly 8 million of which are generated per year in Europe alone.

ELVs and other waste streams such as 'Waste Electrical and Electronic Equipment' (WEEE) contain large quantities of valuable non-ferrous metals which can be profitably recycled and returned to the supply chain - metals such as aluminium, copper, brass, lead, magnesium, nickel, tin and zinc. This has lead to the demand for new sensor technology to economically recover ever high proportions of non-ferrous material (recovery-rate) at ever higher purities of the resulting recyclate (purity rate). At present, the most common approach is handsorting with generally low-paid workers separating the metals by eye using their colour [2]. A more technological solution is dense-media or sink-float systems, where slurries of a specific gravity are created to separate metals according to density. While conceptually simple, the slurries tend to be toxic and must be processed and cleaned to limit environmental damage [3], making cleaner and dryer methods considerably more desirable.

Sensor-based solutions include X-ray transmission and fluorescence (XRT, XRF), laser induced breakdown spectroscopy (LIBS), and optical systems [4]. XRF and LIBS are the goldstandard, commonly used in hand-held form by the industry to analyse different metal types and alloys. However, translating this technology towards an industrial scale sorting solution has proven challenging. Typical plant conveyor belt speeds move the metal fragments at a rate of between $2-3 \mathrm{~m} / \mathrm{second}$ making targeted multiple laser firings with LIBS difficult. Further, the high cost of both technologies makes them uneconomical under in all but the most specialist and high-value lines. Optical systems using colour, and sometimes allied with other sensing technology such as induction, have shown some effectiveness, especially in the separation or stainless steel [4]. Commercial examples include Combisense (Tomra) and KSS system (Steinert). While generally lower cost and easier to scale, optical systems can suffer from indirect lighting, machine vibration, and most significantly given the target industry, the presence of surface contaminants obscuring the fragment colour.

We propose Magnetic Induction Spectroscopy (MIS) as a method to identify classes of non-ferrous metals in mixed waste streams. This approach is a variation of traditional induction-based measurements systems (e.g. metal detectors) wherein we use the secondary magnetic field emitted by an object, in this case a shredded metal fragment, induced by eddy-currents excited by a primary magnetic field over a range of frequencies to obtain a spectra of the object. It is supposed that useful information about the object can be inferred by interpretation of this spectra. Recent applications include the identification of unexploded ordinance [5], [6], [7], characterisation of coinage [8], and physio-chemical properties of agricultural produce [9], [10].

Initially, we investigated a model-based approach to deter- 
mine the conductivity of the metal fragment from geometric data. We developed a fast boundary element solver which uses the thin-skin assumption [11] as a forward model [12]. This proved to be impractical in terms of the solve time required for the rate of material and that only partial 3D geometric information could ever be measured. However in more recent work [13], [14], we were able to demonstrate a surprisingly simpleyet-effective algorithm could be devised to separate copper, aluminium and brass fragments - metals which compose the majority of non-ferrous waste fraction [15], [16] - using only two frequency components of the impedance spectra. Purity and recovery-rates of $\approx 92 \%$ were found using test fragments manufactured in to random shapes cut from known control metal stock. In this paper, we now re-examine our previous work using a new modular multi-frequency induction sensor called the MetalID System and obtain new results with real test samples drawn from commercial shredded waste sorting lines.

\section{A. Theory}

Define $\mathbf{V}_{t x}(\omega)=V_{t x}(\omega) \mathrm{e}^{j \omega}$ as the voltage applied across an excitation coil oscillating at frequency $\omega$. The excitation coil induces and excitation magnetic field. If a metal fragment is exposed to this field, a secondary magnetic field will be induced by the eddy-currents circulating in the fragment. This secondary field can be measured by a second coil (receive coil). The voltage induced in the receive coil by the secondary field is denoted as $\mathbf{V}_{r x}(\omega)=V_{r x}(\omega) \mathrm{e}^{j \omega}$. We define the magnetic induction spectra as the ratio of these two voltages over all frequencies,

$$
\frac{\mathbf{V}_{r x}(\omega)}{\mathbf{V}_{t x}(\omega)}=\frac{V_{r x}(\omega)}{V_{t x}(\omega)}=Z(\omega)=Z^{\prime}(\omega)+Z^{\prime \prime}(\omega) i, \quad \forall \omega
$$

in what follows we will refer to the real component and imaginary component at a frequency $p$ as $Z_{p}^{\prime}$ and $Z_{p}^{\prime \prime}$ respectively.

A typical spectra for a metal fragment is shown in figure 1. The shape is distinctive and common to non-ferrous metal fragments, generally varying only in the proportions and position in frequency along the impedance spectra (e.g. see [17], [18]). The real or in-phase component is shown to be sigmoid shape. As frequency increases the component converges to an asymptote. The imaginary or quadrature component on the other hand shows a curve after an extrema decreasing towards zero as frequency increases.

The shape is intuitively explained by the well-known skindepth effect. As frequency increases, eddy-currents excited in the fragment tend towards the boundary. At high frequencies near the asymptote, the eddy-currents effectively become bound to the surface. Here the secondary magnetic field becomes invariant to the fragment conductivity as the penetration depth is negligible and the magnetic field is reflected back inphase with the excitation.

Our classification algorithm, termed the two Component method or classifier, compares the real component at a frequency close to asymptote which is a function of the geometry of the shape but is independent of conductivity, with an

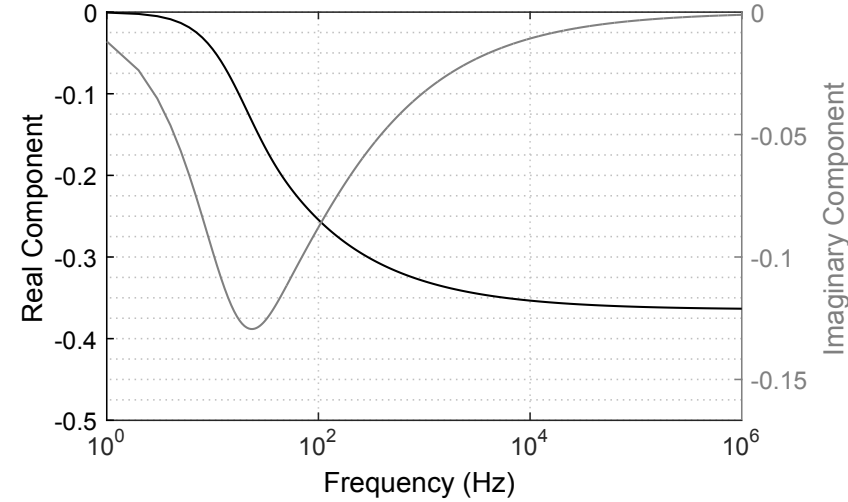

Fig. 1. Typical magnetic induction spectra - derived from a sphere model using [18].

imaginary component at a lower frequency which is a function of both. The aim is to reject the confounding variable of fragment geometry and classify the materials according to their conductivity following a set of reference curves. The approach of being simple, straightforward and transparent, and is practically easy to implement - both in terms of sensordesign and the required computational hardware to be able to operate at milliseconds processing speeds.

\section{METHOD}

\section{A. MetalID System}

The MetalID system, as shown in figure 2, is a new singlechannel variant of the system presented in O'Toole et al. [13] for measuring the magnetic induction spectra of metal scrap fragments as they pass over the top of sensor on a fast moving conveyor belt. It uses a modular design combining a single sensing element with a low-cost embedded Zynq FPGA based signal processing platform (Red Pitaya STEMLAB125$14 \mathrm{~V} 1.0)$ to cover a single channel, but where additional channels can be added across any conveyor belt width by simply repeating the units underneath and across the width of the belt. For typical scrap fragments in the 10-50 mm fraction, the approximate sensitive zone (or channel width) is around $30-50 \mathrm{~mm}$ wide. Therefore, a sensing element would be required every $30-50 \mathrm{~mm}$ of belt width to ensure full coverage. In what follows, we only consider a single channel.

The main components of the system are shown in figure 2 and 4 with an electrical schematic in figure 3. They are (1) a sensing element, (2) bespoke front-end electronics board, and (3) The Red Pitaya processing platform. The sensing element is composed of two solenoids co-incident along their axis placed within an aluminium cylindrical enclosure. The solenoids and their schematic are shown in figure 2 . The inner solenoid consists of two 32 turn coils each wrapped around a ferrite rod. This coil is used to induce an excitation magnetic field a short distance above the sensing element and will be referred to as the excitation coil.

The outer solenoid consists of two 600 turn coils wound in opposing directions and connected in the manner of a gradiometer. This coil measures the resultant secondary magnetic 
field emitted by the metal fragment as it passes over the top of the sensing element. These coils will be referred to as the receive coil. The gradiometer arrangement ensures that the voltage induced in the receive coil by the excitation will be cancelled or minimised. The position of the excite coil with respect to the receive coil may be adjusted using a lead-screw at the base of the sensing element in order to balance the two halves of the gradiometer.

The excite coil is driven by a power amplifier (LT1210, Linear Technologies) using a multi-sine waveform output from the Red Pitaya digital-to-analogue converter (DAC). Currently, the MetalID system is set to simultaneously excite at 3, 9, 15, 30 and $60 \mathrm{kHz}$. However, it can in principle excite at any combination of frequencies between $3 \mathrm{kHz}$ (the fundamental frequency) and $125 \mathrm{kHz}$ (the receive coil resonance) provided that the frequencies are at least $3 \mathrm{kHz}$ apart. As more frequencies spread the excite power, only 5 are chosen for this experiment and only pairs will be used to explore classification following our method in O'Toole et al. [13].

The voltage across the receive coil is measured by a lownoise differential amplifier (AD8249, Analog Devices) and input to the analogue-to-digital converter (ADC) of the Red Pitaya. The ADC and DAC both sample at $125 \mathrm{MHz}$. The Red Pitaya demodulates the measured receive voltage using an FFT block within the FPGA, and processes the stream of demodulated components - a set of real and imaginary components returned at approximately $3 \mathrm{kHz}$ - in to a single set of components for each fragment passing over the sensor using a matched filter. The template of the matched filter is built by summation of the absolute values of the frequency components as the fragment passes and then normalised to the peak value. Thus for each fragment passing over the sensor, a single set of 10 components are returned, i.e. a pair of real and imaginary components for each of the five frequencies. These are recorded for subsequent analysis on a desktop computer

\section{B. Measurement System}

The MetalID system is situated underneath a $100 \mathrm{~mm}$ wide x $1200 \mathrm{~mm}$ long conveyor belt as shown in figure 4 at a depth such that the top of the receive coil is approximately $10 \mathrm{~mm}$ from the top of the belt. Metal fragments are deposited on the belt via a small half-pipe at the feed-end of the conveyor (righthand side of figure 4) which serves to locate the fragments close to the central axis of the sensing element. The pieces are traversed across the sensing element at $\approx 1000 \mathrm{~mm} / \mathrm{second}$ until being discharged into a collection bin at the distal end of the conveyor (left-hand side of figure 4).

\section{Calibration}

The MetalID system is calibrated using a small ferrite cylinder (Ferroxcube, 4B1) with diameter $10 \mathrm{~mm}$ and height $20 \mathrm{~mm}$. The ferrite is placed $5 \mathrm{~mm}$ above the sensing element along its central axis using an acrylic fixture. Calibration parameters are then defined such that $Z_{p}^{\prime}=1$ and $Z_{p}^{\prime \prime}=0$ for all measurement frequencies. The calibration does not align measurements to an absolute value but rather compensates for any phase variations present in the system and provides a common reference between measurements.

\section{Test Samples}

Following O'Toole et al. [13], we will investigate three materials that make-up the majority of commercial non-ferrous waste - aluminium, brass and copper [15]. In total there are 49 samples of aluminium, 100 brass, and 98 copper consisting of varied shapes and sizes, all drawn from commercial shredded metals waste. An exemplar sub-set of theses samples are shown in figure 5. The fragment sizes are approximately between 10 and $100 \mathrm{~mm}$ in size along their longest dimension.

Tests are performed by dropping each fragment in turn along the positioning half-pipe and pushing them down to the moving belt.

\section{RESULTS AND DISCUSSION}

Figure 6 compares the imaginary components at lowfrequencies $(3,9,15,30 \mathrm{kHz})$ with the real component at a high frequency $(60 \mathrm{kHz})$. This is comparable to O'Toole et al. [13] where the imaginary components at 2, 4, 8, and $16 \mathrm{kHz}$ were compared to the real component at $64 \mathrm{kHz}$. In that work, $4 \mathrm{kHz}$ and $8 \mathrm{kHz}$ plots marginally showed the clearest spread of data points, however in our case, no significant difference can be inferred by observation.

In figure 6, we do see regions associated with each individual metal emerging in the order of conductivity, e.g. from the top of the graph we have copper followed by aluminium followed by brass. However, there is significant variability and overlap with neighbouring regions. This is in contrast with O'Toole et al. [13] where the regions where discrete and distinctive, and where a clear trend-line was visible. It is evident that we can only partially replicate the results in our former work when using real scrap metal.

We now explore using this data for classification. We choose the components $\left(Z_{60 k \mathrm{~Hz}}^{\prime}, Z_{3 k \mathrm{~Hz}}^{\prime \prime}\right)$ as features for a two component classifier along the lines specified in section II. Given the similarity of point-spread in figure 6 , we would not expect significant difference if we were to select one of the other pairs in the figure.

In our previous work [13], we fitted a set of logistic functions to the data-set of each metal type as this appeared to closely match the distribution of the data. This is not the case in figure 6 . We will therefore use an ordinary linear regression method for classification. We also choose a binary decision process where a single metal is classified from the whole data rather than attempting to classify all three metals simultaneously. This is in line with commercial sorting systems which typically have two separation bins and where material can be re-fed through the system under different 'modes' to separate the different metal types. For example, in our first test we will extract recovery and purity-rates using the decision (ALU, $\overline{A L U}$ ) from the whole of the data as if the separation mode had been set to extract aluminium. In our second test we consider the decision $(\mathrm{BR}, \overline{\mathrm{BR}})$ from the whole of the data, and so forth. 

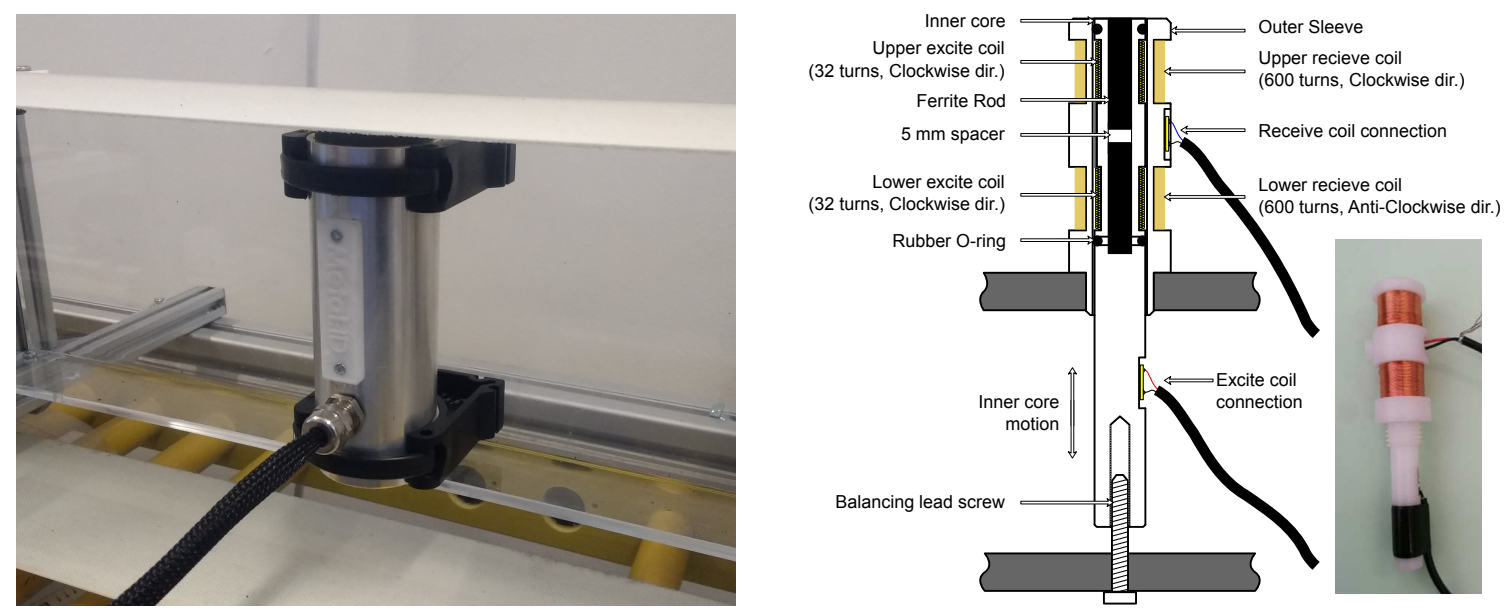

Fig. 2. MetalID Sensing element situated under a single channel conveyor belt (left). Internal schematic of the sensing element showing the excite and receive and coils [13] (right).

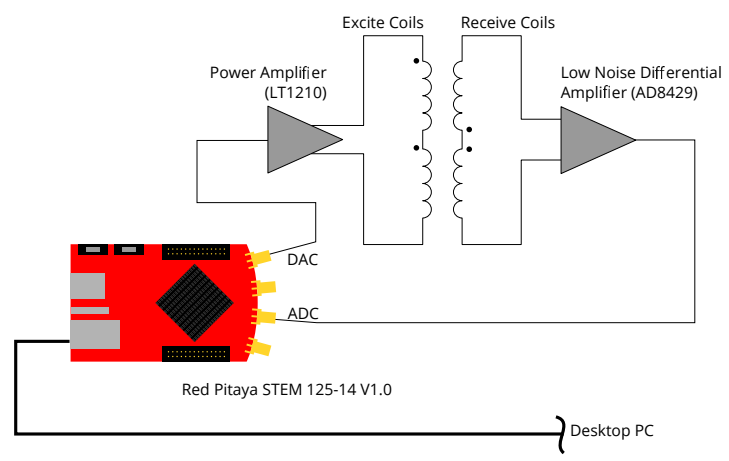

Fig. 3. Electrical Schematic of the MetalID System

We define a simple binary classification algorithm as follows: A fragment $a$ with measured components $\left(Z_{60 k \mathrm{H} z}^{\prime}, Z_{3 k \mathrm{~Hz}}^{\prime \prime}\right)$ belongs to a class $C$ if,

$$
w_{C, 1} Z_{60 k H z}^{\prime}+w_{C, 2} Z_{3 k H z}^{\prime \prime}<t
$$

where $t$ is some chosen threshold and $\left(w_{C, 1}, w_{C, 2}\right)$ are a pair of coefficients which maximise the between class variance while minimising the between class variance. The class $C \in\{\mathrm{ALU}, \mathrm{BR}, \mathrm{CU}\{$ is selected according to which metal we wish to extract.

For each metal type, we consider two metrics: (1) recoveryrate which is the proportion of that material recovered from the whole, and (2) purity-rate which is the proportion of recovered material which actually is that material. Formally,

Rec.-rate $=T p /(T p+F n), \quad$ Purity-rate $=T p /(T p+F p)$,

where $T p, F n, F p$ are True-positives, False-negatives, and False-positive respectively.

In figure 7 we show the receiver operating characteristic (ROC) curves comparing recovery and purity rates for each metal by varying the threshold parameter $t$. The highest performance overall was for copper with an $\approx 80 \% / 80 \%$ recovery-rate/purity-rate ratio. This is followed by brass with $\approx 80 \% / 75 \%$. The aluminium classification is disappointing with a ratio of $\approx 80 \% / 55 \%$ indicating a high proportion of false positives. No metal was found to have close to $100 \%$ purityrate even at the expense of a low recovery-rate. This is in contrast to $\approx 92 \% / 92 \%$ found in O'Toole et al. [13] and is substantially below reported rates for other technologies such as 90-97\% for X-ray techniques, $80-97 \%$ for LIBS and $86-95 \%$ for optical methods [19]. Evidently, the manufactured sample set featured in this work was not able to sufficiently capture the rich geometry of the scrap fragment metal fragments and the effect this geometry has on the induction response. There may also be a significant range of alloys present within the sample set with a matching range of conductivities - some potentially overlapping with other metal types. While we have shown that materials of simple shapes can be successfully classified using two component classification, the challenge remains in how to adapt and augment this method in order for it to address the complexities and variability present in a real scrap sorting line.

\section{CONCLUSION}

Magnetic induction spectroscopy is a powerful technique for analysing the electrical properties of objects by measuring the scattering of a secondary magnetic field. We have proposed that this approach has utility in determining classes of different non-ferrous metals in the recycling industry in a manner which is practical, capable of high-throughput, and requires modest and relatively inexpensive technology to implement.

In our past work, we were able to that MIS could be used to classify copper, brass and aluminium with a simple algorithm using two frequency components on test samples manufactured from known stock. The present work re-explored this algorithm and found that we could not replicate the initial success when testing on real scrap samples. We were able to show similarities between the past and present work when comparing the two frequency components, such as the ordering and pattern ordering of the metal classes within the features space. However, the coherency and the distinctiveness of the 




Fig. 4. The single channel experimental test system.

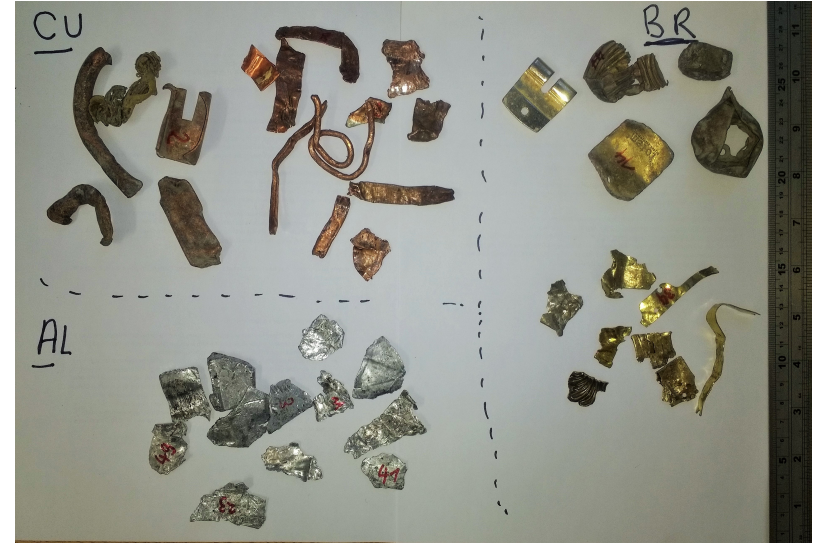

Fig. 5. Examples of typical test scrap metal pieces. Copper pieces are shown in the top left, brass on the right, and aluminium in the bottom left.

different regions and the close logistic-function-shaped trendlines of the original could not be replicated. Furthermore, we were not able to achieve the impressive recovery and purity-rates reported in the original work, in this case finding only modest performance statistics for brass and copper, and generally poor statistics for aluminium. It is supposed that some mixture of physical, electrical or geometric variability of the fragments in a typical scrap stream is beyond the classification facility of this simple two-component method.

However, our method still holds promise. The statistics for copper and brass while modest, are still not insignificant, especially given the inexpensive measurement instrumentation relative to the high value of these metals. We can conceive of certain scenarios where our method works in concert with over sensor modalities in order to leverage their economic value. For instance, X-ray-based separation technologies and float-sink tanks struggle to separate brass and copper due to their almost identical densities. In contrast, our approach is relatively successful at discriminating these two metals. Our method could be used as priming stage, providing a lowaccuracy gross-sorting method to filter batches for slower but more accurate sorting technologies such as LIBS.

Finally, our research continues to investigate new feature sets derived from the magnetic induction spectra to improve classification ability. In this work, we continued with our pro- posed two component classifier as such a simple algorithm has many advantages in terms of sensor design and computational burden at high-throughput. However, we note the plethora of available features yet to be explored, such as multiple frequency components, temporal and spatial data, derivatives and spectral shape parameters, and the magnetic polarisability tensor [20]. It is clear from the fundamental theory that the magnetic induction spectra of an object encodes much information about its physical and electrical properties, but analytical approaches of greater sophistication and intelligence are demanded to prise a useful advantage in the recovery of non-ferrous metals.

\section{REFERENCES}

[1] "Directive 2000/53/ec of the european parliament and of the council of 18 september 2000 on end-of life vehicles," Official Journal of the European Communities, vol. 43, 2000.

[2] G. Gaustad, E. Olivetti, and R. Kirchain, "Improving aluminum recycling: A survey of sorting and impurity removal technologies," $R e$ sources, Conservation and Recycling, vol. 58, pp. 79 - 87, 2012.

[3] W. L. Dalmijn and T. P. R. De Jong, "The development of vehicle recycling in europe: Sorting, shredding, and separation," JOM, vol. 59, no. 11, pp. 52-56, Nov 2007.

[4] M. Kutila, J. Viitanen, and A. Vattulainen, "Scrap metal sorting with colour vision and inductive sensor array," in International Conference on Computational Intelligence for Modelling, Control and Automation and International Conference on Intelligent Agents, Web Technologies and Internet Commerce (CIMCA-IAWTIC'06), vol. 2, Nov 2005, pp. $725-729$.

[5] O. A. A. Rehim, J. L. Davidson, L. A. Marsh, M. D. O’Toole, D. W Armitage, and A. J. Peyton, "Measurement system for determining the magnetic polarizability tensor of small metal targets," in 2015 IEEE Sensors Applications Symposium (SAS), April 2015, pp. 1-5.

[6] L. A. Marsh, J. L. Davidson, M. D. O'Toole, A. J. Peyton, D. Ambras, D. Vasic, and V. Bilas, "Spectroscopic identification of anti-personnel mine surrogates from planar sensor measurements," in 2016 IEEE SENSORS, Oct 2016, pp. 1-3.

[7] O. A. Abdel-Rehim, J. L. Davidson, L. A. Marsh, M. D. OToole, and A. J. Peyton, "Magnetic polarizability tensor spectroscopy for low metal anti-personnel mine surrogates," IEEE Sensors Journal, vol. 16, no. 10, pp. 3775-3783, May 2016.

[8] J. L. Davidson, O. A. Abdel-Rehim, P. Hu, L. A. Marsh, M. D. OToole, and A. J. Peyton, "On the magnetic polarizability tensor of us coinage," Measurement Science and Technology, vol. 29, no. 3, p. 035501, 2018. [Online]. Available: http://stacks.iop.org/0957-0233/29/i=3/a=035501

[9] M. D. O'Toole, L. A. Marsh, J. L. Davidson, Y. M. Tan, D. W. Armitage, and A. J. Peyton, "Rapid non-contact relative permittivity measurement of fruits and vegetables using magnetic induction spectroscopy," in 2015 IEEE Sensors Applications Symposium (SAS), April 2015, pp. 1-6.

[10] - "Non-contact multi-frequency magnetic induction spectroscopy system for industrial-scale bio-impedance measurement," Measurement Science and Technology, vol. 26, no. 3, p. 035102, 2015. [Online]. Available: http://stacks.iop.org/0957-0233/26/i=3/a=035102 

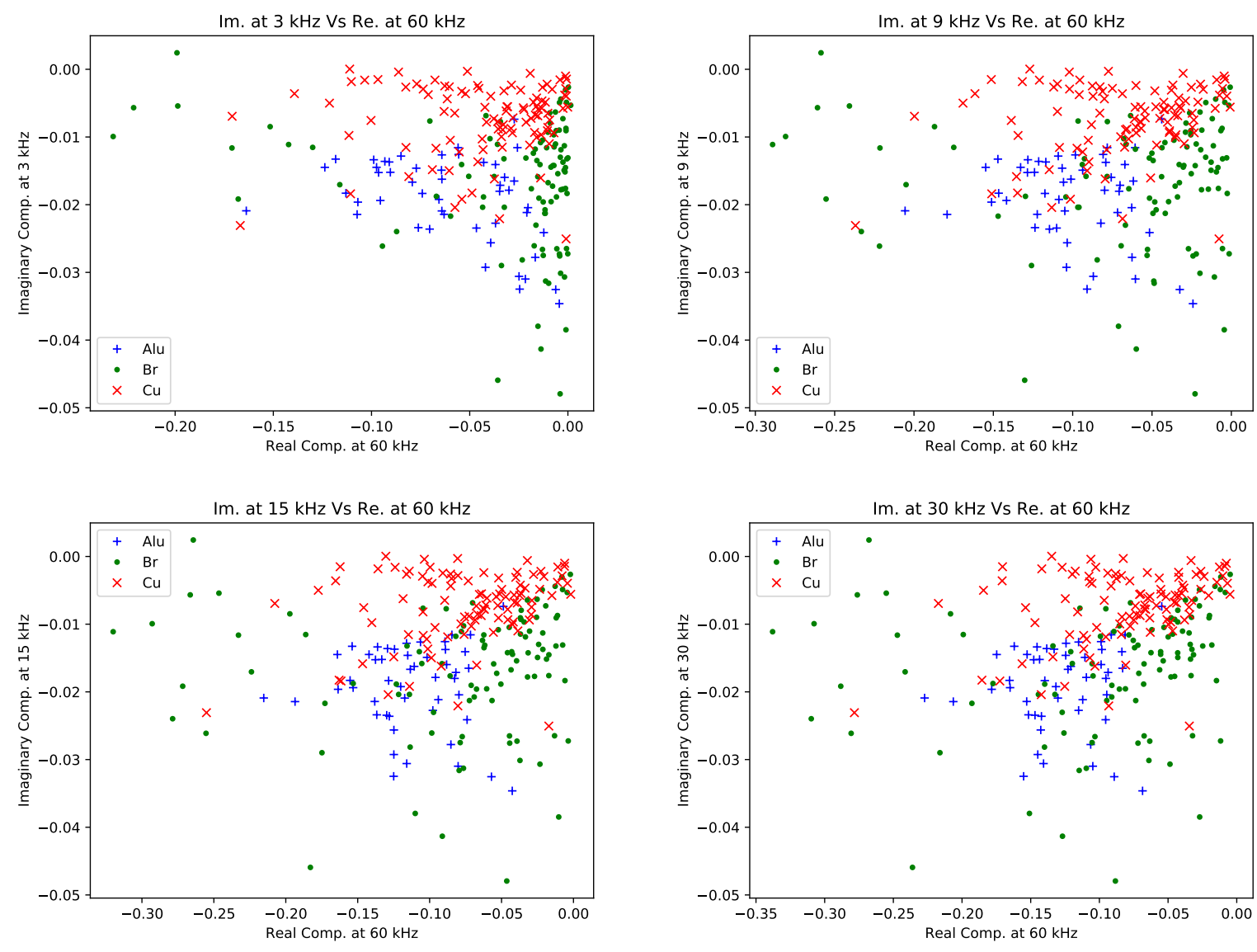

Fig. 6. Comparison of the imaginary component of the spectra $Z^{\prime \prime}$ at frequencies $3 \mathrm{kHz}$ (top left), $9 \mathrm{kHz}$ (top right), $15 \mathrm{kHz}$ (bottom left) and $30 \mathrm{kHz}$ (bottom right) with the real component Z' at $60 \mathrm{kHz}$.

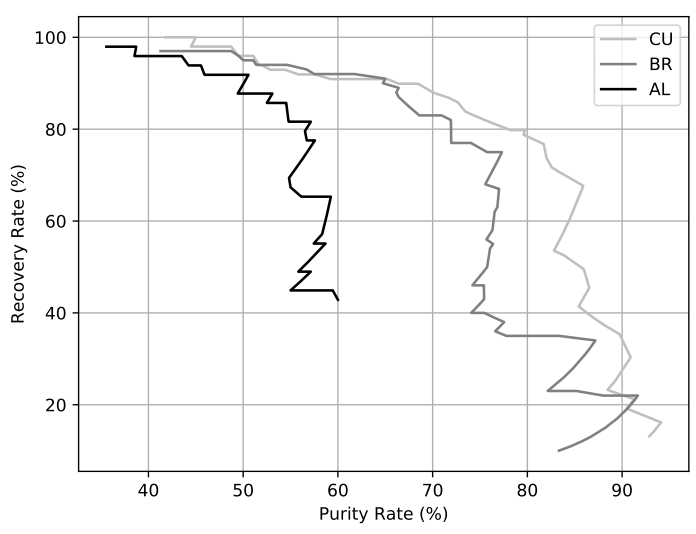

Fig. 7. Receiver operating characteristic (ROC) curve comparing Recovery and Purity rates.

[11] K. Sun, K. O'Neill, F. Shubitidze, S. A. Haider, and K. D. Paulsen, "Simulation of electromagnetic induction scattering from targets with negligible to moderate penetration by primary fields," IEEE Trans. on Geoscience and Remote Sensing, vol. 40, no. 4, pp. 910-927, Apr 2002.

[12] M. D. O’Toole, J. L. Davidson, L. A. Marsh, W. Yin, and A. J. Peyton, "Evaluation of the thin-skin approximation boundary element method for electromagnetic induction scattering problems," in 2016 IEEE Sensors Applications Symposium (SAS), April 2016, pp. 1-6.

[13] M. D. OToole, N. Karimian, and A. J. Peyton, "Classification of nonferrous metals using magnetic induction spectroscopy," IEEE Transactions on Industrial Informatics, vol. 14, no. 8, pp. 3477-3485, Aug 2018.

[14] M. D. O'Toole, N. Karimian, and A. J. Peyton, "Fast classification of non-magnetic metal targets using eddy-current based impedance spectroscopy - to be published," in 2017 IEEE SENSORS, Oct 2017.

[15] F. Margarido, R. N. Santos, F. Durão, C. Guimarães, C. Nogueira, P. C. Oliveira, F. Pedrosa, and A. M. Gonçalves, "Separation of non-ferrous frations of shredded end-of-life vehicles for valorising its alloys," in MMME'14-International Conference on Mining, Material and Metallurgical Engineering, 2014, pp. 77-1.

[16] I. of Scrap Recycling Industries, ISRI Scrap Specifications, Circular 1994: Guidelines for Nonferrous Scrap, Ferrous Scrap, Glass Cullet, Paper Stock, Plastic Scrap. Institute of Scrap Recycling Industries, 1994.

[17] J. R. Wait, "Some solutions for electromagnetic problems involving spheroidal, spherical, and cylindrical bodies," Res. NBS (Math. and Mathematical Physics) B, vol. 64, pp. 15-32, 1959.

[18] _ "A conducting permeable sphere in the presence of a coil carrying an oscillating current," Canadian Journal of Physics, vol. 31, no. 4, pp. 670-678, 1953

[19] S. P. Gundupalli, S. Hait, and A. Thakur, "A review on automated sorting of source-separated municipal solid waste for recycling," Waste Management, vol. 60, pp. 56 - 74, 2017, special Thematic Issue: Urban Mining and Circular Economy.

[20] P. D. Ledger and W. R. B. Lionheart, "Understanding the magnetic polarizability tensor," IEEE Transactions on Magnetics, vol. 52, no. 5, pp. 1-16, May 2016. 